\title{
LA AUTOEDUCACIÓN PERMANENTE EN UN CONTEXTO SOCIOTÉCNICO
}

\author{
Gonzalo Vázquez Gómez \\ Universidad Complutense de Madrid
}

\begin{abstract}
RESUMEN: La educación permanente es un proceso de autoconstrucción basado en los principios pedagógicos de la educabilidad, la educatividad y la autonomía personal. El proceso de construcción humana implica un proceso de aprendizaje y de formación a lo largo y ancho de la vida a fin de conseguir el "bienestar" (la vida buena) individual y comunitario. Esta concepción de la educación permanente incluye la consideración de nuevos espacios de aprendizaje como el de la ciudad cognitiva y nuevas posibilidades como la constituida por la existencia de los cursos abiertos. Dentro del enfoque de la formación de las capacidades se estudian las posibilidades y riesgos del aprendizaje autorregulado como una estrategia eficaz en el actual contexto socio-técnico y de globalización tecnológica. Finalmente, se examina el potencial de riesgo que supone la aportación de la analítica del aprendizaje y de la inteligencia artificial (IA) a la educación para cuya superación se reclama que el proceso de educación permanente esté basado en una sólida formación humana.
\end{abstract}

PALABRAS CLAVE: educación permanente, educación a lo largo y ancho de la vida, ciudad educadora, enfoque de las capacidades, contexto sociotécnico, cursos abiertos, IA, educación básica.

\section{LIFELONG SELF-EDUCATION IN A SOCIOTECHNICAL CONTEXT}

\footnotetext{
ABSTRACT: Permanent education is a process of self-construction based on the pedagogical principles of educability, educativity and personal autonomy. The process of human construction implies a process of lifelong and lifewide learning and training in order to achieve individual and community "wellbeing". This conception of continuing education includes the consideration of new learning spaces such as that of the cognitive city and new possibilities such as the open courses. Within the approach of capabilities building, the possibilities and risks of self-regulated learning are studied as an effective
} 
strategy in the current socio-technical and technological globalization context. Finally, the risk potential of the contribution of learning analytics and artificial intelligence (Al) to education is examined; and it is concluded that the process of permanent education in a socio-technical context must be based on a solid human formation conceived as basic education.

KEYWORDS: Permanent education, Lifelong and lifewide education, Educating city, Capability approach, Socio-technical context, Open courses, Al, Basic education.

Recibido: 10/12/2019

Aceptado: 05/02/2020

Correspondencia: Gonzalo Vázquez Gómez. Universidad Complutense de Madrid, Facultad de Educación, c/ Rector Royo Villanova, s/n 28040 Madrid. E-mail: gvazquezg@gmx.net.

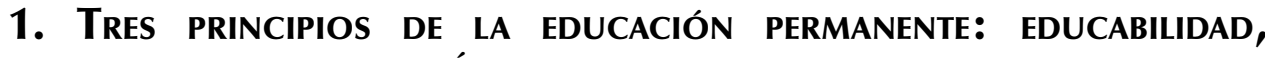 EDUCATIVIDAD Y AUTONOMÍA}

El proceso educativo descansa en el principio básico de la educabilidad de la persona. Según nos ha dicho la antropología filosófica y pedagógica, el hombre es un animal inacabado que necesita de la relación con el medio físico y social para acabar de serlo. Este principio básico de la educabilidad da origen a la educación permanente: mientras el hombre lo es, es educable. En palabras de Kant, en Pedagogía, el hombre es la única criatura que ha de ser educada pues "únicamente por la educación el hombre puede llegar a ser hombre" (2003, p. 31).

En estrecho correlato con este primer principio de la educabilidad existe un segundo principio, el de la "educatividad" que se manifiesta en un imperativo moral: el mandato del "edúcate a ti mismo", "sé tú mismo" a través de la educación, de la autoeducación. De nuevo Kant apela a este principio cuando dice que "la Providencia ha querido que el hombre deba sacar el bien de sí mismo y le habló, por decirlo así: ‘¿Entra en el mundo!; yo te he provisto de todas las disposiciones para el bien. A ti te toca desenvolverlas..."' (2003, p. 34). De la interacción entre ambos principios, el de la educabilidad y el de la educatividad, se deduce, como corolario, que todo individuo es siempre educando y educador de sí mismo. Para lograr que cada uno llegue a construir su sí mismo es preciso que la educación, entendida como educación básica, construya la autonomía cognitiva y moral del sujeto y con ella la capacidad suficiente para autoconstruirse como persona a lo largo de toda la vida. La autonomía personal en el proceso de aprendizaje es el tercer principio que, conjuntamente con la educabilidad y la educatividad, posibilita la autoconstrucción humana.

El nacimiento y desarrollo inicial de la educación permanente ha estado ligado a la educación de adultos, a los procesos de alfabetización, incluida la alfabetización elemental (Maíllo, 1969). En la actualidad, el empeño de la educación de adultos 
está puesto en la capacitación cognitiva y moral del individuo y en su contribución al desarrollo de la comunidad. Se exige hoy a la educación que contribuya al desarrollo solidario y sostenible en el espacio y en el tiempo, así como al logro de la calidad de la vida personal y colectiva. Se concede tanta importancia a la educación permanente dentro de los sistemas educativos contemporáneos que puede afirmarse, como se hace en la exposición de motivos de la LOGSE en España (1990), que todo el sistema educativo descansa en torno en el principio de la educación permanente, principio confirmado en los textos de las posteriores leyes educativas.

El problema que se nos plantea, y que constituye el objeto de este trabajo, es el de identificar las metas y estrategias de la educación permanente en la actualidad. Y lo haremos en este tiempo en el que confluyen tres fenómenos característicos: la reconstrucción del espacio y tiempo en los sistemas culturales y productivos, la globalización económica y el desarrollo de la tecnología (ahora bajo el formato de la tecnología digital).

Consideramos que la autoeducación, enfocada como un proceso de autoorganización y autocontrol dentro del nuevo contexto cultural, social y tecnológico (contexto socio-técnico), es una estrategia básica para el desarrollo personal y comunitario. Este proceso de autoeducación se basa en la autonomía personal y asegura una multiplicidad de actuaciones que expresan la pluralidad propia de la acción humana (Arendt, 2015). Gracias a esta continua construcción autónoma el sujeto de la educación, no solo se construye a sí mismo dentro de unas condiciones que se le han dado en un contexto espacial y temporal determinados, sino que es capaz de configurar nuevos contextos de aprendizaje y desarrollo personal. Para lograrlo necesita la base de una sólida formación básica sin la cual el individuo no puede recorrer el itinerario de su proceso de educación permanente.

\section{La eduCACIÓN A LO LARGO Y ANCHO DE LA VIDA}

El concepto de educación permanente se ha extendido en dos dimensiones, en el tiempo y en el espacio. Ese cambio se hizo patente hace ya medio siglo y ha tomado una nueva forma en los últimos veinte años. En una primera instancia se concibió que la función del aprendizaje y de la educación supera los límites de la escolaridad, incluso más allá de la educación obligatoria y de la educación postsecundaria y superior. Se reconocía así la entidad del "aprendizaje a lo largo de toda la vida" considerado como un "proceso por medio del cual los individuos adquieren conscientemente una educación formal o informal a través toda su vida para su desarrollo personal y profesional" (Thesaurus ERIC, 2019).

En los últimos años del siglo pasado aparecen diferentes documentos de organismos internacionales (OCDE, Comisión de las Comunidades Europeas) que cambian la perspectiva de análisis de las funciones y extensión de los sistemas educativos. Por una parte, se reclama una educación permanente para todos a lo largo de toda la vida, una educación de la que nadie quede excluido (en términos actuales diríamos que la educación permanente debe ser inclusiva). Llama la atención que esta demanda a los sistemas educativos a la universalidad de la educación continuada, más allá 
de los límites de la escolaridad obligatoria, se hiciera al mismo tiempo que todavía no se había cumplido en su plenitud una de las metas del nuevo milenio (ahora formuladas de otro modo dentro de los Objetivos para el Desarrollo Sostenible con horizonte temporal en el 2030), la de que todas las niñas tuvieran asegurada al menos una educación básica de cinco años de escolaridad.

Es posible reconocer, además, otra contraposición, en este caso entre participación en la educación formal en su nivel terciario (más allá de la educación secundaria) y la transferencia desde el lugar propio donde se dan los aprendizajes. Por una parte, es cierto que las tasas de educación terciaria están creciendo de manera continua (los últimos datos disponibles, en noviembre de 2019, de la OCDE, referidos al año 2017, cifran en el 44,5\% el promedio de la población entre 25 y 34 años con estudios de esa educación terciaria, y el 27,2\% para la población de 55 a 64 años). Pero, por otra, la participación en el aprendizaje formal tiende a declinar con la edad en los países desarrollados en los que los individuos de edad superior a los 55 años de grupos socioeconómicos con capacidades superiores tienden a implicarse en programas y actividades autodirigidas o de grupos de aprendizaje informal sobre la base de una sólida experiencia educativa previa (Government Office for Science, 2017).

Junto a la extensión de la educación de los individuos a lo largo de todo el curso de la vida, esto es, en el tiempo, se ha producido un ensanchamiento de su campo de acción que afecta al espacio. El Memorándum sobre el Aprendizaje Permanente, del año 2000, de la Comisión de las Comunidades Europeas incluye dos propuestas innovadoras. Por una parte, se reclama la acción convergente de los tres ámbitos del aprendizaje (formal, no formal e informal). Por otra, se requiere que, junto al aprendizaje a lo largo de la vida, se desarrolle un aprendizaje "a lo ancho de la vida" que comprometa al conjunto de espacios en los que se desarrollan los individuos. Desde que Reichman hablara por primera vez en 1986 de "lifewide learning" esta expresión se ha convertido en un lugar común dentro de las prácticas, de la política y de la teoría de la educación permanente (Jackson, 2012). Un tópico que se ha convertido en utopía pedagógica bajo la fórmula de la educación de calidad para todos a lo largo y ancho de la vida (Vázquez, 2002).

En estos últimos años, y gracias a este cambio de perspectiva, se concibe que los sistemas educativos se extienden afuera y más allá de la escuela y de la escolaridad obligatoria. Esta idea se vincula con el concepto de ciudad ya nos refiramos a esta, bien en términos de ciudad del aprendizaje, bien como ciudad cognitiva, e incluso finalmente como ciudad educadora. La ciudad como espacio de conocimiento es un campo estudiado desde la óptica de la ciencia cognitiva en la geografía, la sociología, la economía y la pedagogía, entre otras disciplinas, según una perspectiva interdisciplinar. Desde una perspectiva más amplia, el concepto de ciudad se vincula con el de región de aprendizaje entendida como "una región en la que todos los agentes colaboran para atender necesidades de aprendizaje específicas y adoptar soluciones conjuntas para problemas comunes" (Comisión de las Comunidades Europeas, 2000, p. 39).

Esta corriente de investigación y de práctica política conoció un gran impulso a principio de los años noventa del siglo pasado. Entonces las ciudades con represen- 
tación en el Primer Congreso Internacional de Ciudades Educadoras, celebrado en Barcelona en 1990 (congreso que conoció las aportaciones de los profesores Sanvisens y Colom sobre la ciudad educadora y la pedagogía urbana). Las ciudades participantes recogieron en la Carta inicial "Los principios básicos para el impulso educativo de la ciudad", principios que abarcan el derecho a una ciudad educadora, el compromiso de esta con la satisfacción de ese derecho y el compromiso, por fin, de prestar un servicio integral a las personas. Dentro de estos servicios integrales a las personas se incluye que "el municipio deberá garantizar información suficiente y comprensible e incentivar a sus habitantes a informarse. Considerando el valor que supone seleccionar, comprender y tratar el gran caudal de información actualmente disponible, la ciudad educadora facilitará recursos al alcance de todos" (Carta de las Ciudades Educadoras, 1990, principio 19). Esta tendencia de los sistemas educativos desde los años noventa ha dado lugar a la creación de la Red Mundial de Ciudades del Aprendizaje de la UNESCO (GNLC).

Se aprecia una progresiva preocupación de la educación permanente por este nuevo espacio de la educación, el de la ciudad, espacio en el que confluyen el derecho a la información con el compromiso con los aprendizajes cívicos y la construcción de la ciudadanía (cfr. Touriñán, 2019. "Dónde está la educación", Educación para el desarrollo, a propósito de los derechos de la cuarta generación). En la metáfora de la ciudad educadora se expresan las relaciones de complementariedad y de substitución y alternancia de la educación formal y no formal, de sendos ámbitos de la educación que progresivamente se encaminan hacia la unificación de los espacios educativos (Colom, 2005; 2016).

Esta nueva forma de concebir el espacio de la ciudad como territorio del aprendizaje supone un cambio de perspectiva, desde la educación como respuesta reactiva a las necesidades específicas que se dan aquí y ahora (una cierta forma de reeducación), hasta un aprendizaje continuo, prospectivo y proactivo. En consecuencia, la ciudad se configura como un espacio de "glocalidad", de compromiso entre lo global y lo local (Touriñán, 2004) y de contribución de los espacios ciudadanos al incremento de la riqueza cognitiva, y por lo tanto económica, de los países y de las regiones (Longworth, 2003, pp. 93-120: "El territorio del aprendizaje eterno"). Los estudios económicos y los análisis econométricos han apreciado y medido progresivamente la contribución de la educación, incluidos los procesos de aprendizaje no formal e informal e innovador, a la riqueza inmaterial y material de los países y de las regiones. Así la economía digital mundial, que implica procesos de innovación tecnológica, representaba en 2016, según el Banco Mundial, un valor del 15,5 \% del producto interno bruto (PIB) mundial y se estimaba que esta cifra llegaría al $25 \%$ en menos de una década (Banco Mundial, 2019).

Tamaña riqueza tiene, claro está, una dimensión ética y adquiere, por lo tanto, una condición cognitivo-moral que expresa el potencial de construcción humana (individual y social) de la ciudad, en su dimensión local y universal. Este potencial tiene una doble polaridad pues, junto a las posibilidades de enriquecimiento, el desarrollo de las ciudades ha originado algunos riesgos en la homogeneidad de las regiones en las que se insertan. Es preciso preguntarse sobre la posibilidad de que convivan 
escuelas que aspiran a ser integradoras dentro de ciudades y de regiones segmentadas. Basta con analizar la localización física de los mapas cognitivos de una misma ciudad (nodos de información, redes o conjuntos ("clusters") industriales, centros de aprendizaje, museos y bibliotecas, centros escolares y hasta resultados de pruebas internacionales del tipo de PISA, STEM, etc. de acreditación), sobre todo en megaciudades, para advertir que la variabilidad interna dentro de una misma ciudad es superior a la variabilidad respecto de otros territorios. Dicho de otro modo: hay tercer y cuarto mundo, no ya lejos, ni siquiera alrededor (en "la periferia", en su sentido más etimológico y formal), de la ciudad sino en el seno de un mismo espacio urbano. Resulta necesario, pues, proceder al enriquecimiento cognitivo de la ciudad mediante la herramienta de la educación básica y la estrategia de la educación de todos a lo largo de la vida como medios para el enriquecimiento de todos.

Debemos cuestionarnos los costes de toda índole (económicos, sociales, políticos y educativos) que acarrean las mega-ciudades sobre los territorios colindantes, cuestión que, más allá del oportunismo y actualismo político, se ha denunciado desde hace mucho tiempo por parte de la geografía humana y económica que ha denunciado que la ciudad pueda convertirse en "un paisaje de exclusión" (García Ballesteros, 1995). Es preciso que la práctica de la concentración urbana y la extensión de la acreditación universal nos sirva para confirmar más el aprendizaje de calidad de todos y no solo para señalar el éxito o fracaso de algunos (Longworld, 2003). En una visión sistémica de la educación, la única inteligente, el fracaso de algunos, ya sea bajo la forma de falta de capacidades básicas, ya de la incapacidad para construir el propio proceso de educación permanente, debe verse como fracaso del sistema, tanto en la versión interna del medio, como en el medio circundante.

Dentro de esas capacidades básicas adquiere una importancia especial la capacidad para la educación permanente que implica una aptitud bastante y actitud abierta hacia el aprendizaje a lo largo y ancho de la vida así como una disposición básica positiva hacia la innovación. La inclinación positiva hacia esta no es solo una condición humana en general, como algo dado que viene impreso en la condición humana, sino que es una variable construible desde la educación y las políticas sociales, económicas y de ordenación del territorio. De aquí que la sociología, la geografía y la economía, entre otras disciplinas, se ocupen de la ciudad y de las regiones como espacios de innovación. Un buen ejemplo se encuentra en las conferencias bienales de Geografía de la Innovación que se vienen celebrando desde 2012 (en Saint-Étienne, Utrecht, Toulouse y Barcelona; y que tendrá lugar en su quinta edición en la ciudad noruega de Stavanger a fines de enero de 2020) y en las que participan especialistas en la política y en las cuestiones relativas de la dimensión espacial de los planes y programas de innovación tecnológica y social.

En definitiva, el cambio de mirada sobre las relaciones entre educación y espacio nos permite ver que el espacio de aprendizaje y educativo sobrepasa con mucho los muros escolares y que el espacio (sea físico, social y ciudadano, o tecnológico; real o virtual) constituye un ámbito de aprendizaje para el crecimiento personal y comunitario. Vemos, al mismo tiempo, el espacio como un ámbito para que el sistema educativo se comprometa con la transferencia del aprendizaje mediante el 
aprendizaje-servicio y la transferencia de conocimiento, función más exigible a la Universidad bajo la fórmula de la "tercera misión" pero que compromete a la totalidad del sistema.

\section{Educarse en Un CONTEXTo SOCIO-TÉCNICO}

Los estudios actuales sobre la educación tecnológica y de los hábitos de consumo de los procesos y productos de la tecnología de la información nos confirman que la tecnología ha adquirido un carácter trivial y ubicuo; trivial en tanto que ordinario y común, ubicuo por cuanto que no hay lugar que no esté penetrado por la tecnología en su doble trayectoria de aportación y de recogida de información.

Antes decíamos que nuestra conducta era en mayor medida de recogida, de "bajada" de información mucho más que de "subida", de aportación; el común de los individuos son mucho más consumidores de información que generadores de ella. Ahora ya hemos pasado de la sospecha a la experiencia comprobada de que, cada vez que recogemos información de la red, estamos aportando información de nosotros mismos, incluso de nuestra identidad, de lo que se deduce una consecuencia supresora de la libertad respecto del futuro de nuestro curso vital: en alguna medida, nuestro futuro educativo dentro de un proceso de desarrollo continuo está ya escrito por la huella digital que firmamos cotidianamente con nuestra participación en los sistemas de información. La educación básica y permanente debe capacitar a los sujetos para que ejerzan el necesario autocontrol sobre la huella de la generación y consumo de información.

Lo que ocurre con el espacio socio-técnico sucede con todo espacio pues, según nos han dicho García del Dujo y Muñoz al proponer una pedagogía del espacio, este es un elemento constitutivo del individuo quien puede ser caracterizado como un "sujeto espacial" (2004). Además de este carácter común con toda relación hombre-espacio, en el caso concreto del espacio tecnológico se dan otras características como la derivada del carácter impositivo de la tecnología, de una tecnología que, no solo impregna todas las esferas de la vida pública (cosa que según estos mismos autores ya se observa en todo fenómeno espacial), sino que invade ya la vida privada de los individuos y de los grupos.

De este carácter ubicuo e invasivo de la tecnología se deriva que una de las misiones de la educación tecnológica ha de ser la de recuperar la condición de sujeto en el par hombre-máquina para lo cual es obligado que la educación capacite a las personas para dominar y dirigir su propio aprendizaje en una variedad de contextos, y singularmente en el contexto socio-técnico, a lo largo de su vida (Sharples, 2000). En fin, se trata de recuperar las dimensiones de autonomía y comunicación propias del entorno socio-técnico entendido como proceso de aprendizaje en red basado en la investigación que combina el aprendizaje individual y el cooperativo y que facilita oportunidades para interactuar con otros, bien sea mediante la tecnología, bien en relaciones cara a cara (Seel, 2012).

Una de las características más problemáticas del aprendizaje en un contexto socio-técnico es la de la relación antagónica entre asimilación y adaptación. Algu- 
nos estudios hechos en contextos de organizaciones innovadoras aportan evidencia a favor de un aprendizaje y de un comportamiento basado en una adaptación autónoma de los procesos de innovación (Hasgall y Ahituv, 2018). Esta actitud es propia de los Ilamados "trabajadores fractales", trabajadores que pueden caracterizarse por cinco notas: poseer, dejar huella y transmitir un estilo propio en su forma de actuar, mostrar una alta sensibilidad ambiental, saber incorporar la información, implicarse personalmente en la red social y, por fin, mostrar una estructura de actitudes positivas hacia la integración de la tecnología de la información en la organización, actitudes, claro está, con sendas dimensiones perceptiva, comportamental y emocional

En este mismo estudio se apunta una conclusión relevante respecto del enfoque más eficaz de control. Para llegar a configurar este tipo de práctica en la vida activa, con sujetos que intercambian energía con su contexto, es preciso que en los estadios iniciales de la experiencia informal y del aprendizaje más o menos formal en la escuela se eviten objeciones a la asimilación de la tecnología bajo la forma de un control físico e inmediato por parte de los educadores (Hasgall y Ahituv). De aquí se deduce una consecuencia implícita sobre el papel del proceso de autocontrol dentro de la educación permanente. Desde luego, no se aprende únicamente en situaciones de control extrínseco, continuo y formal, aunque tampoco en lugares donde no esté presente alguna forma de control externo como condición necesaria para que se produzca una regulación inteligente de la acción, según nos dirá Dewey.

El aprendizaje informal de la tecnología supone el reconocimiento del potencial del aprendizaje experiencial, de la relación entre educación y experiencia formal de aprendizaje cuyo fundamento encontramos en la obra de Dewey (Experience and Education, 1938) quien destaca la relación de continuidad e interacción de lo que se aprende con la experiencia previa y futura del sujeto De aquí que los sistemas productivos evalúen cada vez con mayor interés los aprendizajes previos, de carácter frecuentemente informal, acumulados por los interesados en formar parte de las organizaciones. Este interés es más acusado respecto de los individuos adultos porque poseen un mayor caudal de experiencia previa y porque cuentan, en consecuencia, con disposiciones más arraigadas respecto de cómo aprender (estilos de aprendizaje, actitudes hacia el trabajo cooperativo y la innovación, etc.).

Situados entre la elección entre asimilación meramente mecánica de la información, sobre todo en situaciones de innovación, y la adaptación en procesos de aprendizaje a lo largo de toda la vida, la mejor respuesta se encuentra en la adaptación dinámica de los cambios. Se confirma así que es más inteligente la conducta de aquellos agentes y sistemas que protagonizan y promueven procesos denominados por Piaget como "equilibración", esto es, procesos que aseguran el intercambio continuo de energía y, en última instancia, la pervivencia del sistema, del sujeto que aprende de una manera "equilibrada" a lo largo de todo su curso vital.

El aprender en contextos socio-técnicos participa de las condiciones y requisitos de toda cognición situada, es decir, del aprendizaje que tiene lugar y actúa en relación con el medio ambiente en el que tiene lugar. Este tipo de cognición implica la participación activa del aprendiz, así como su interacción social y la interacción 
entre los nuevos aprendizajes y el conocimiento y la experiencia previa y contemporánea de los sujetos que participan en el proceso (McClintock, Streibel y Vázquez, 1993).

Más específicamente, el aprendizaje propio de las personas adultas en contextos de aprendizaje permanente es el denominado "aprendizaje transformador" entendido como el "aprender mediante una reflexión crítica de las propias experiencias, asunciones, creencias, sentimientos y perspectivas mentales con el propósito de revisar o construir nuevas interpretaciones" (ERIC. Thesaurus, 2019). Esta manera de concebir el aprendizaje descubre la relación entre aprendizaje y pensamiento, sobre todo de pensamiento reflexivo y crítico, de experiencia vivida y de discurso racional. Es posible pensar que en el futuro el aprendizaje de los sistemas socio-técnicos se basará en una tecnología cada vez más inteligente, y al mismo tiempo más social. Eso significa, según Steghofer (2017), que la relación entre el sujeto y la tecnología implicará la conducta humana, la toma de decisiones e interacciones no deterministas, la cultura, la ética y por encima de todo los valores (a menos que, como nos advierte el autor, "todo esto quede irrevocablemente cambiado por la propia tecnología"). Este riesgo solo podrá afrontarse desde una discusión pública sobre los límites de la tecnología y, en cualquier caso, a partir de la capacidad de discernimiento del sujeto, de una capacidad que le permita actuar de forma autónoma frente a los estímulos, condiciones y requisitos propios de los desarrollos tecnológicos.

\section{Posibilidades, límites y amenazas de la educación Permanente: el APRENDIZAJE AUTORREGULADO DESDE LA PERSPECTIVA DE LA PEDAGOGÍA DE LA PERSONA}

Nuestra era se ha caracterizado como la era del aprendizaje, y nuestros tiempos como los del aprender con un horizonte ilimitado. Esta consideración del proceso de aprendizaje, esa concepción borrosa de la frontera entre la educación formal e informal, está poniendo en cuestión el sentido mismo de la Escuela, del conocimiento meramente escolar y del control extrínseco de lo aprendido. Al propio tiempo, existe una corriente principal de investigación, de teoría y práctica educativa a favor del aprendizaje profundo, del aprendizaje crítico y reflexivo, características que solo se pueden dar a partir de la fundamentación de la educación en la autonomía del sujeto y desde una solución armoniosa entre el autocontrol y la intervención y el control extrínseco.

Un proceso de formación permanente ha de cumplir con tres exigencias: ser un proceso autodirigido, configurarse como un proceso de autorregulación, y como un proceso que, originándose en una situación específica, vaya más allá de las circunstancias concretas en la que se produce la experiencia concreta de aprendizaje. En primer lugar, decimos que el aprendizaje educativo es un aprendizaje autoconstruido, concepto de aprendizaje que se tomado cuerpo teórico en la psicología humanista de la segunda mitad del siglo XX pero que se encarna en la más vieja tradición humanista sobre la causación de la educación (Bouchard, 2011; 2012). Este autor considera que quien aprende de manera autodirigida es un sujeto activo, energético, libre y consciente que habitualmente elige sus metas y la dirección que quiere im- 
primir a su comportamiento y que no actúa simplemente empujado y arrastrado por fuerzas internas inconscientes.

El aprendizaje construido por uno mismo implica un proceso continuo de autorregulación. La capacidad personal para la regulación del propio proceso implica el dominio de ciertas competencias básicas. Sin embargo, la autorregulación no es propiamente una habilidad mental o una destreza de ejecución académica; más bien es un proceso autodirectivo por medio del cual los aprendices son capaces de transformar sus disposiciones mentales en habilidades académicas (Zimmerman, 2002). La propuesta de este autor nos advierte que la autorregulación no pone el énfasis en métodos de aprendizaje aislados del contexto social, sino en la iniciativa y la perseverancia personal de los sujetos. Los aprendices según este enfoque son capaces de activar, modificar y mantener prácticas de aprendizaje, tanto en contextos sociales como de estricta individualidad.

El aprendizaje autorregulado se caracteriza por su complejidad y estructuración. Incluye todo un conjunto ordenado de funciones de alto nivel tales como la cognición situada, la metacognición, la resolución de problemas y la motivación. La cognición situada se apoya en la teoría de la cognición social que reconoce que el contexto físico, técnico y social estimula y modifica los procesos de aprendizaje, pero que también es modificado gracias a la interacción con el sujeto que aprende (McClintock, Streibel y Vázquez, 1993; Liang y Yang, 2017).

El autocontrol es el núcleo funcional y teórico de la autorregulación del aprendizaje en los sistemas. El control así entendido es necesario para el buen funcionamiento del sistema, y más en el caso de los sistemas inteligentes como es el caso de los sistemas educativos y de los individuos que aprenden de manera continuada. Se concede tal importancia al autocontrol que se ha podido decir que "la finalidad ideal de la educación es la creación de la capacidad de autocontrol", como se afirmaba ya en Experiencia y Educación. Sin embargo, nos advierte Dewey en esta obra sobre los riesgos de reducir toda forma de control al autocontrol del sujeto que aprende a través de la experiencia. Nos previene que no existe garantía alguna de que, al suprimir el control externo, no estemos saliendo de una situación mala para caer en otra peor dado que "los impulsos y deseos que no están ordenados por la inteligencia quedan bajo el control de las circunstancias accidentales" para concluir que "una persona cuya conducta se controla de este modo solo tiene en el mejor de los casos una ilusión de libertad" (Dewey, 1938, p. 64).

La educación permanente desde la pedagogía de la persona requiere distinguir el pensar del mero aprender. Dialogar y pensar en un contexto exige ir más allá del problema específico y de las circunstancias inmediatas que lo rodean toda vez que todo contexto tiene una dimensión extra-situacional (Goodwin y Duranti, 1992) sin la cual no cabe conversación alguna. Precisamente porque la educación permanente es un proceso continuo, con un desde y un hacia, es necesario que la experiencia focal y local de lo inmediato en el tiempo y en el espacio se integre y proyecte en el pensar humano como función trascendente. Dewey se preguntaba sobre los distintos sentidos del pensar y concluyó que, más de allá de entenderlo como lo que tenemos en la mente, como creencias sobre hechos, o como lo que nos Ileva a aceptar algo 
como razonablemente probable o improbable, el pensar en su sentido más profundo es aquello que cuestiona las bases y consecuencias de las creencias, incluidas las consecuencias operativas (Dewey, 1910, pp. 2-5). Esta perspectiva sobre las funciones del pensar da sentido a la educación permanente, sobre todo en el contexto actual de globalización y cambio permanente.

El cambio continuo y la variedad contextual constituyen sendas amenazas a la individualidad y a la consistencia de la identidad del sujeto (riesgo del que nos previene Conrad, 2007). Por ello resulta preciso y urgente construir una teoría de la educación permanente desde la perspectiva de la pedagogía de la persona. Así se lo ha planteado Wheelahan (2007) quien, siguiendo el pensamiento de Barnett (2004), se ha preguntado qué tipo de currículum, de cualificaciones y de pedagogía necesitamos con vistas a un futuro incierto. Se propone aquí que, dada la situación de cambio permanente, es necesario cuestionarse qué tipo de conocimiento ha de procurarse dentro del y a partir del currículum. Se apunta a un tipo de conocimiento que vaya más allá del conocimiento del tipo I (conocimiento fragmentado de las cosas) e incluso del tipo II (conocimiento desde la perspectiva interdisciplinar que facilita la identificación y resolución de problemas). Es necesario orientar los sistemas educativos y los procesos de formación permanente hacia un conocimiento del tipo III, un conocimiento que se dirige "nada menos que a la transformación del ser humano" (Barnett, 2004, pp. 256-257). Este es un conocimiento vagamente estructurado, provisional y que se confronta con incertidumbres y dilemas y con problemas o cuestiones abiertas cuyo tratamiento provoca una intensa implicación personal. Queda aquí cuestionada la validez del enfoque de las competencias genéricas, competencias que no serían válidas sino para los tipos I y II de conocimiento, por lo que, en última instancia, nos llevarían a la trayectoria típica del callejón sin salida para enfrentarnos con alguna posibilidad de supervivencia a los problemas de un mundo cada vez más complejo y abierto.

Ni el currículum fragmentado, basado todavía en el libro impreso y compuesto por asignaturas, ni siquiera el currículum interdisciplinar aseguran, desde este enfoque de la pedagogía de la persona, la capacidad personal para pensar y para hacer frente a un cambio cada vez más acelerado y profundo. Esta incapacidad amenaza la estabilidad del sujeto y sus posibilidades de entender el mundo y de entenderse con él, lo que genera estados de insatisfacción, de inseguridad y de ansiedad por parte de los individuos y de las comunidades de aprendizaje. La necesidad de esta forma de pensar supone trascender la idea de que el hombre solo se realiza plenamente más allá del conocimiento y de la acción entendida como alteración de la naturaleza y como fabricación de nuevos artefactos desde el trabajo humano (con lo que se consuma como animal laborans; Arendt, 2015). Esta dificultad se agranda cuando los procesos de aprendizaje tienen lugar con la mediación tecnológica, como es el caso más frecuente hoy en día; en tal caso sigue vigente el pensamiento de Bruner cuando afirma (en On Knowing) que, en nuestro tiempo, las exigencias de la tecnología amenazan la libertad del individuo para crear imágenes del mundo que nos sean satisfactorias en su sentido más profundo (Bruner, 1965).

Se deduce, en consecuencia, la necesidad de que el proceso de educación permanente ha de fundamentarse en una sólida capacitación básica, una cultura común 
y una buena respuesta a la articulación entre las humanidades y el sistema de ciencia y tecnología. Esta formación básica se reclama en el Libro Blanco sobre la educación y la formación. Enseñar y aprender. Hacia la sociedad cognitiva (Comisión de las Comunidades Europeas, 1995) cuando demanda que todos los jóvenes europeos tengan una sólida cultura general para saber responder a los tres choques motores de la sociedad: los de la información, de la mundialización y de la civilización científica y técnica. Según este Libro Blanco la primera respuesta a esta crisis consiste en poseer una cultura general ya que "existe un riesgo de que la sociedad europea se divida entre los que pueden interpretar, los que sólo pueden utilizar y los que quedan al margen en una sociedad que les da asistencia: es decir, entre los que saben y los que no saben" (Ibíd., p. 8).

Como es bien sabido, las capacidades de análisis profundo e interpretación forman parte de las competencias cognitivas de alto nivel (del nivel sexto de las apreciadas en las pruebas del PISA). Significa esto que los individuos que no han alcanzado el dominio de esas capacidades al concluir la educación obligatoria quedan muy limitados para participar en los procesos de educación permanente y para la participación activa en los procesos de construcción de la ciudadanía. La expectativa de que la tecnología iba a reducir las tasas de analfabetismo está lejos de verse, pues, satisfecha; muy al contrario, estamos advirtiendo que solo disminuye el analfabetismo elemental pero que al propio tiempo se ha constituido en un nuevo espacio fértil para la generación de nuevos analfabetismos que limitan la construcción de la ciudadanía consciente y justa.

En efecto, saber no consiste solo en conocer y es mucho más que hacer: implica ser capaz de interpretar los problemas de nuestro mundo para la existencia individual y colectiva (Vázquez, 2016). Sin una buena base de cultura general que preste soporte a la competencia tecnológica se incrementan las posibilidades de que la tecnología se convierta en "una nueva forma de exclusión social" para los colectivos menos familiarizados con ellas para hacer frente a los riesgos potenciales de ciberseguridad y protección de datos. Así se ha denunciado recientemente por autoridades del Banco de España con ocasión del "Día de la Educación financiera" (7 de octubre de 2019). El caso más característico dentro de este contexto socio-técnico-financiero es el de las "fintech" pero se puede extender a las relaciones con la administración, el comercio electrónico o la educación electrónica ("e-administración", "e-comercio", "e-educación"). Quien no haya asimilado una buena formación tecnológica, quien no posea una adecuada competencia tecnológica (según la propuesta de Sarramona, 2016), sobre la base de una sólida formación humana y cultural, pasará a verificar la previsión de Kaku, hace más de treinta años, cuando nos decía que "la sociedad podría convertirse en una nación de «ricos» y «pobres» de información" (1998, p. 160).

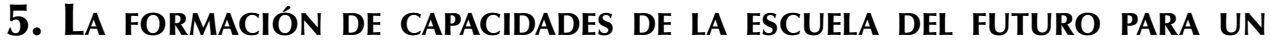 PROCESO DE FORMACIÓN PERMANENTE}

Concluimos que la educación permanente requiere una educación básica de calidad para todos. Ahora bien, debemos preguntarnos todavía cuál es la misión del sistema educativo para fundamentar ese proceso de educación a 
lo largo de toda la vida. La respuesta, provisional, que goza de un mínimo consenso, abraza dos principios: el de la substitución del enfoque de las competencias por las capacidades; y el de la revisión del concepto de escuela superando el enfoque de la escuela adaptativa.

El enfoque de las capacidades ha sido objeto de trabajo desde la antropología, la filosofía política y la economía (con decisivas aportaciones de Arendt, Nussbaum y Sen). Constituye un cuerpo teórico que implica, según Robeyns en la Stanford Encyclopedia of Philosophy, dos principios normativos: "la libertad para conseguir el bienestar tiene una importancia moral básica, y la libertad para alcanzar ese bienestar debe comprenderse en términos de capacidades de la gente, de sus oportunidades reales para hacer y ser aquello que valoran como razonable" (Robeyns, 2016). Según este enfoque, las capacidades están configuradas como posibilidades efectivas, de libertades, para ser y hacer lo que se desea a fin de alcanzar una calidad de vida saludable (de vida buena). Preguntarse hoy por la calidad de la educación es preguntarse por una dimensión de la calidad de vida que implica "por igual" a todos. Ahora bien, según la doctrina de las capacidades, la cuestión no reside en fijar estándares de calidad, sino en identificar aquellas capacidades consideradas básicas para sobrevivir y para escapar de la pobreza y de otras privaciones que limitan el desarrollo humano (Nussbaum y Sen, 1996; Sen, 2005).

Lo que se juega en este escenario del enfoque de las capacidades es algo cualitativo, y no estándares más o menos altos de desarrollo; se trata de identificar cuáles son los límites de la pobreza por debajo de los cuales no se puede afirmar que hayamos alcanzado la condición de justicia conforme a la dignidad humana. Dentro del concepto de pobreza reconoce la Unesco dos modalidades, la pobreza absoluta y la relativa. La absoluta es de índole económica y se expresa en relación con los ingresos, per cápita y día en dólares, necesarios para satisfacer las necesidades básicas (se considera que el umbral de un dólar por día es el que delimita la pobreza absoluta). En cambio, la pobreza relativa hace referencia a la calidad de vida y significa que quienes se encuentran en ella no han alcanzado o han perdido las condiciones para participar de una manera efectiva y justa en los bienes de la comunidad. Pues bien, el concepto de pobreza relativa ha adquirido en nuestro tiempo un carácter poliédrico; dentro de ella se incluye también la cara de la pobreza educativa (Unesco, 2019). Y, por lo tanto, podemos concluir que en nuestro tiempo la pobreza educativa afecta a los individuos y comunidades que no cuentan con niveles mínimos de educación básica y de capacitación para la educación permanente.

En segundo lugar, para asegurar la posibilidad de la educación permanente es preciso superar el modelo actual que concibe la escuela como el espacio donde tiene lugar el aprendizaje de una manera todavía fragmentada, aprendizajes que son sometidos a un control retrospectivo y confirmatorio. Sobre este punto ya existe mucha doctrina, aunque todavía poca experiencia sometida a un riguroso control teórico y de verificación. Ya en los últimos años del siglo pasado podemos encontrar voces autorizadas que reclaman un cambio hacia adelante en el modo como concebir el aprendizaje escolar. Así, McClintock (1993) emparentando con una vieja tradición 
pedagógica, aboga por la educación liberal en la cual el estudiante desarrolla la capacidad de adquirir conocimientos posteriores, destrezas y comprensión por sí mismo: "Esta autodirección responsable es la marca de una persona autónoma. Una persona educada liberalmente, confrontada con un nuevo reto, sabe encontrar las fuentes, tiene suficiente confianza intelectual para sentir lo que necesita saber para poder empezar, puede juzgar lo que es relevante" (McClintock,1993; en McClintock, Streibel y Vázquez, p. 121).

En efecto, lo que se dirime aquí es cómo ha de entender el aprendizaje una escuela que ha de capacitar para la educación permanente. Colom (1997) dice al respecto que es preciso crear un nuevo sistema educativo diseñado como espacio de creación de conocimiento. Aclara el autor que en términos tecnológicos el cambio estribaría en "superar nuestra escuela actual, asentada en el feed-back, en la capacidad de adaptación a la realidad [actual] para lograr una escuela que concibiese la educación como la inclusión en los educandos de la capacidad de feed-before; o sea, del control proyectivo" (Colom, 1997, p. 12).

Tamaño cambio de perspectiva implica, no solo el necesario cambio en la institución escolar, sino incluso en el alumno-educando de modo que le permita enfrentarse con eficacia a las situaciones futuras. Pues de eso se trata: de concebir la educación como la formación de capacidades para comprender el mundo, para darle sentido a la experiencia hombre-mundo, y para contribuir a su mejora para toda la humanidad.

\section{Una posibilidad actual PaRa la aUtoeducaCión PeRmanente: los CURSOS ABIERTOS}

La educación permanente está conociendo un nuevo desarrollo bajo la fórmula de los cursos abiertos. La idea básica se expresa de manera muy sencilla tal como se lee en el sitio OCW del Instituto Tecnológico de Massachussets (M.I.T.): "la idea es simple: publicar todos nuestros materiales en la red y hacerlos ampliamente disponibles a todos (D. K. P. Yue, Professor, MIT School of Engineering)". Estos cursos implican una manera revolucionaria de concebir el aprendizaje y el aprendiz: "tanto si usted es un estudiante, un profesor o simplemente una persona curiosa que quiere aprender, encontrará un mundo de visión e inspiración en el MIT OpenCourseWare" (OCW.MIT.edu).

Por su parte, la Universidad de Columbia puso en marcha en 2012 Coursera a partir de la decisión de dos profesores (D. Koller y A. Ng) de compartir su conocimiento y su saber hacer con todo el mundo con idéntica motivación: "la de hacer posible que cualquiera pudiera aprender en red en un tiempo limitado el conocimiento cuyo aprendizaje les hubiera llevado la vida entera en situaciones ordinarias de clase".

Las plataformas creadas se han puesto a disposición de las universidades más reputadas de Estados Unidos y de todo el mundo. Las universidades contribuyen al aprendizaje con su plataforma y con miles de cursos de naturaleza muy variada de humanidades, ciencias sociales y tecnología, y de otros campos. Esas plataformas incluyen conferencias grabadas, materiales y tareas con autoevaluación y 
revisión por pares y foros de discusión; en todo caso, los cursos están graduados por niveles, desde el nivel de principiante hasta el más avanzado, y se ofrecen con una estimación del nivel requerido de entrada y la carga de tiempo para su superación. Algunos de los cursos son gratuitos; en cualquier caso, para obtener una acreditación formal de los aprendizajes alcanzados deben abonarse unas tasas específicas.

En la plataforma edX se encuentran más de 2400 cursos, más de veinte millones de alumnos de todo el mundo con más de setenta millones de inscripciones (Plataforma edX, 2019) datos tomados en https://www.edx.org/ el 19 de noviembre de 2019). Muchos de estos cursos se ofrecen con la organización, servicio y acreditación de varias universidades e instituciones (por ej.: por el MIT y Harvard University, el Banco Interamericano de Desarrollo, el Instituto Tecnológico de Monterrey, IBM y otras universidades europeas, iberoamericanas, asiáticas, etc.).

Como puede apreciarse por el escaso tiempo desde la puesta en marcha de estos cursos y la numerosísima oferta de estos cursos masivos abiertos y en red (MOOC: Massive Online Open Courses) es necesario preguntarse por las características del aprendizaje que se genera en ellos, cuestión sobre la que todavía falta una contrastación suficiente. Bouchard $(2011 ; 2012)$ se ha preguntado sobre el alcance de una pedagogía cuando el profesor no está presente y ha identificado cuatro dimensiones que deben controlarse en el aprendizaje autorregulado: algorítmica, conativa, semiótica y económica a fin de que sea rentable en términos personales. La dimensión algorítmica cubre la secuenciación, el ritmo, la formulación de objetivos y la identificación de recursos; la conativa incluye las intenciones y motivos para aprender; la semiótica afecta a la índole de los materiales (impresos, en red, etc.) y su interacción con el estudiante; y, por último, la dimensión económica viene dada por el valor intangible del conocimiento, por su valor de mercado, por los costes de oportunidad por haber renunciado a otras acciones de estudio o trabajo, por el lucro cesante generado, etc. (Bouchard, 2011).

Más recientemente, y cuando ya se contaba con la experiencia de algunos años de los cursos MOOC, se ha publicado el trabajo de Terras y Ramsay (2015) sobre los fundamentos psicológicos del aprendizaje autorregulado que permite apuntar los posibles puntos críticos del trabajo con estos cursos. Estos puntos son de tres tipos: bien los relativos a las dimensiones socio-cognitivas del estudiante; bien de carácter motivacional, emocional y de compromiso intelectual; bien a su nivel de alfabetización digital y su capacidad de autorregulación de los procesos de aprendizaje (Terras and Ramsay, 2015).

Estos estudios van estableciendo una base, que será necesario consolidar, sobre los fundamentos psicológicos, pedagógicos y tecnológicos de estos cursos abiertos y masivos. Uno de los aspectos que será menester investigar en el futuro es el de la interacción entre el individuo que sigue el curso y el conjunto, la red social, de los participantes; y también la índole y los efectos de la interacción inconsciente entre el hombre y la máquina (Wakkary, Desjardins y Hauser, 2015). 


\section{Educarse a lo largo de toda la vida: ¿Cuán Permanente ha de Ser LA EDUCACIÓN DEL INDIVIDUO?}

La educación permanente parte del presupuesto de la educabilidad y la educatividad humana y de que el hombre siempre puede dar más de sí, según la pretensión pascaliana. En consecuencia, resulta oportuno preguntarse por los límites de ese proceso educativo. Esta cuestión nos devuelve al problema de la relación entre calidad de educación y calidad de vida y el logro del bienestar para lo que debemos recordar que, según el enfoque de las capacidades, la calidad del bienestar no se corresponde necesariamente con el logro de altos estándares de ejecución, sino con la satisfacción de las necesidades propiamente humanas que permiten a los individuos tener un conciencia digna de sí Esta distinción afecta al modo como se evalúan las capacidades lo que, según Sen, no debe hacerse mediante el contraste de los logros de bienestar de una persona, sino con la libertad de lograr esa libertad de modo que le permita lograr combinaciones de funcionamientos humanos valiosos en el ámbito de lo que la propia persona pueda y quiera ser, llegar a ser (Sen, 1998; 2005).

Desde esta base, podemos deducir que el límite de todo el proceso educativo se identifica con la conquista de la individualidad y la excelencia (Rodríguez Neira, 2016). Esto implica una solución armoniosa a la contraposición entre la apertura constante a la realidad y a su conocimiento y el equilibrio y mantenimiento de la propia identidad. De otro modo, el individuo no podrá sobrevivir a "la sobresaturación de informaciones, el imperio de lo efímero, de la moda y del afán de novedades [que] dibujan un mundo permanentemente cuestionado y sometido a transformación, un mundo evanescente" (2016, p. 72; cfr. también del mismo autor T. R. Neira, 1999. La cultura contra la escuela). La educación permanente es la condición de desarrollo de la persona; las ofertas cotidianas y masivas de información, procesadas de manera acrítica, constituyen una amenaza radical a la libertad y autonomía del proceso de autoconstrucción de la excelencia personal.

Los procesos de participación social en distintos campos, del consumo de productos, de servicios de salud y de educación, están generando una cantidad de datos, de información, que arrojan un doble balance, positivo y negativo. Por una parte, es posible que "la influencia del ordenador y de Internet hayan hecho aumentar, en vez de disminuir, nuestra libertad de expresión y nuestro acceso a la información" (Kaku, 1998, p. 160), pero no debemos ignorar los peligros reales y entre ellos que la informática arroje a la indigencia a millones de personas, así como "la amenaza a las libertades civiles (intimidad, censura y escuchas ilegales)" (Ibíd., ibíd.).

Este problema puede alcanzar dimensiones gigantescas en el caso de la información generada por los millones de participantes en los cursos MOOC con sus respectivos datos acerca del conjunto de usuarios, de sus itinerarios de formación, de las fuentes consultadas, de los datos de buenas y malas prácticas en el aprendizaje, etc. Las mismas máquinas que facilitan el acceso a la información y el crecimiento personal de los sujetos albergarán por mucho tiempo una parte sustantiva de lo que leen, escriben y piensan; tanto de los contenidos, como de los procesos de aprendizaje, tiempos, ritmos, estilos cognitivos, aplicaciones, síntesis personales y actitudes y valores generados a lo largo de su formación. 
Comprobada la naturaleza y magnitud del problema se está formando hoy una conciencia colectiva sobre la gravedad de sus efectos en el campo educativo. En noviembre de 2019 se ha publicado un número monográfico del British Journal of Education Tecnology sobre la analítica del aprendizaje y la inteligencia artificial (IA), cuestión que se examina desde tres perspectivas, la política, la pedagógica y la de las prácticas educativas. La perspectiva política se adopta para advertir la ansiedad generalizada que se está produciendo ante el temor de que los algoritmos y la IA puedan aplicarse a los datos sobre la educación; desde la óptica pedagógica se plantea la discusión sobre los efectos que los algoritmos y la IA puedan generar sobre la evaluación cuantitativa de los aprendizajes en perjuicio de una visión más comprensiva y cualitativa de los acontecimientos culturales; por último, se preguntan los autores sobre la naturaleza de los diseños de aprendizaje y del currículum que podrán en marcha los profesores cuando se expanda el uso de los algoritmos y la inteligencia artificial (Shum y Luckin, 2019).

Esta es una cuestión abierta a la que se deberá prestar mayor atención en los próximos años dentro del actual y futuro contexto socio-técnico y de la globalización tecnológica y económica. Es un problema abierto a la discusión interdisciplinar en la que la pedagogía también debería tener una palabra que decir. Por ahora, habremos de preguntarnos por qué niños, jóvenes y adultos, y algunos ancianos, están permanentemente encadenados a unos artefactos tecnológicos que les cargan con el peso de la información, que no del conocimiento y del pensar en profundidad (Vázquez, 2016).

Una educación permanente que abarque la competencia tecnológica y que esté anclada en una consistente formación humana, en la que prime el espíritu crítico y el ejercicio de la libertad, es una herramienta eficaz para afrontar este problema con algunas posibilidades de supervivencia.

\section{REFERENCIAS BIBLIOGRÁFICAS}

Arendt, A. (2015). La condición humana. Barcelona: Paidós.

Banco Mundial (2019). Desarrollo digital. (www.bancomundial.org).

Barnett, R. (2004). Learning for an unknown future. Higher Education Research and Development 23(3), 247-260.

Bouchard, P. (2011). Pedagogy without a teacher: What are the limits. International Journal of Self-Directed Learning, 7(2), 2-20.

Bouchard, P. (2012). Self-directed Learning and Learner Autonomy. En N. M. Seel (Ed.), Encyclopedia of the Sciences of Learning (pp. 2997-3000). Boston, MA: Springer.

Bruner, J. (1965). On knowing. Essays for the Left Hand. N. York: Atheneum.

Carta de las Ciudades Educadoras (1990). http://www.edcities.org

Colom, A. J. (1990). La pedagogía urbana, marco conceptual de la ciudad educadora. En. J. Fernando y S. Morell (Coords.), I Congreso Internacional de Ciudades Educadoras (pp. 115-130). Barcelona: Ayuntamiento de Barcelona. 
Colom, A. J. (1997). La regionalización de la educación como tecnología cognitiva. Teoría de la Educación, 9, 7-19.

Colom, A. J. (2005). Continuidad y complementariedad entre la educación formal y no formal. Revista de Educación, 338, 9-22.

Colom, A. J. (2016). La educación en la escuela y fuera de ella. ¿Una diferenciación obsoleta? En Grupo SI(e)TE Educación, Repensar las ideas dominantes en educación. Santiago de Compostela: Andavira.

Comisión de las Comunidades Europeas (2000). Memorándum sobre el Aprendizaje Permanente. Bruselas: Comisión de las Comunidades Europeas.

Conrad, D. (2007). The pedagogy of confession. Exploring identity and the online self (pp. 193-203). En Osborne, Houston, and Toman, The Pedagogy of Lifelong Learning. London: Routledge.

Dewey, J. (1910). How we think. Boston: D. C. Heath \& Co., Publishers. (Hay traducción española: (2007). Cómo pensamos: nueva relación entre pensamiento y proceso educativo. Barcelona: Paidós). Recuperado de http://www.gutenberg.org/ ebooks/37423

Dewey, J. (1938). Experience and Education. (Hay traducción española: Experiencia y educación. Madrid: Biblioteca Nueva, 2004). N. York: Touchstone Rockefeller Center.

Duranti, A., y Goodwin, Ch. (Eds.) (1992). Rethinking context: Language as an interactive phenomenom. Cambridge (UK): Cambridge University Press.

ERIC (2019). Thesaurus. Recuperado de http://www.eric.ed.gov

García Ballesteros, A. (Coord.) (1995). La ciudad: objeto de estudio pluridisciplinar. Madrid: Ed. Oikos-Tau.

García del Dujo, Á. y Rodríguez, J. M. (2004). Pedagogía de los espacios. Esbozo de una horizonte educativo para el siglo XXI. Revista Española de Pedagogía, 62 (228), 257-278.

Geografía de la Innovación [Geolnno] (2019). Recuperado de http://www.edcities.org

Government Office for Science (2017). Future of Skills and Lifelong Learning. London: Crown.

Hasgall, A. y Ahituv, N. (2018). Implementing continuous adaptation to technology innovation in complex adaptive organizations. The Journal of High Technology Management Research. http://doi.org/10.1016/j.hitech.2018.04.004.

Jackson, N. (2012). Lifewide Learning: History of an idea. Chapt. 1 in Lifewide Learning, Education \& Personal Development (e-book). Recuperado de http://www. lifewideebook.co.uk/conceptual.html

Kaku, M. (1998). Visiones. Cómo la ciencia revolucionará la materia, la vida y la mente en el siglo XXI. Madrid: Debate. 
Kalz, M. (2015). Lifelong learning and its support with new technologies. En N. KJ. Smelser, y P. B. Baltes (Eds.), International Encyclopedia of the Social and Behavioral Sciences. Oxford: Elsevier.

Kant, I. (2003). Pedagogía. Traducción de L Luzuriaga y J. L. Pascual del alemán (1803). (3ª ed.). Edición de Mariano Fernández Enguita. Madrid: Akal.

Kay, J. y Kummerfeld, B. (2019). From data to personal user models for lifelong, life wide learners. British Journal of Educational Technology, 50(6), 2871 2884.

Liang, L. y Yang, M. Z. (2017). Understanding Self-Regulated Learning within an EFL Online Formative Assessment Module. Open Journal of Social Sciences, 5(8), 312-320.

Longworth, N. (2003). El aprendizaje a lo largo de la vida. Ciudades centradas en el aprendizaje para un siglo orientado hacia el aprendizaje. Barcelona: Paidós Ibérica.

Low, R. y Jin, P. (2012). Self organized learning. En N. M. Seel (Ed.), Encyclopedia of the Sciences of Learning. Boston, MA: Springer.

Maíllo, A. (1969). Educación de adultos. Educación permanente. Madrid: Editorial Escuela Española.

McClintock, R., Streibel, M. y Vázquez, G. (1993). Comunicación, tecnología y diseños de instrucción. La construcción del conocimiento escolar y el uso de los ordenadores. Madrid: MEC-CIDE.

Neira, T. R. (1999). La cultura contra la escuela. Barcelona: Ariel.

Nilson, L. B. (2013). Creating Self-Regulated Learners. Strategies to Strengthen Students' Self-Awareness and Learning Skills. Sterling (Virginia): Stylus Publishing.

Nussbaum, M. C. y Sen, A. (Comps.) (1996). La calidad de vida. México D. F.: Fondo de Cultura Económica.

Osborne, M., Houston, M. y Toman, N. (2007). The Pedagogy of Lifelong Learning. London: Routledge.

Plataforma edX, (2019). https://www.edx.org/

Robeyns, I. (2016). The Capability Approach. En Edward N. Zalta (Ed.), The Stanford Encyclopedia of Philosophy (Winter 2016 Edition). Recuperado de https://plato. stanford.edu/entries/capability-approach/

Rodríguez Neira, T. (2016). La excelencia como ideal educativo (pp. 67-77). En Grupo SI(e)TE, Repensar las ideas dominantes en educación. Santiago de Compostela: Andavira.

Rushby, N. y Surry, D. (Eds.) (2016). The Wiley Handbook of Learning Technology. Hoboken, NJ: John Wiley and Sons. 
Sarramona, J. (2016). La competencia tecnológica. Un desafío para la cultura y la escuela del siglo XXI (pp. 243-258). En A. Colom y C. Lisón C. (Eds.), Antropología, cultura y educación. Valencia: Tirant lo Blanch.

Seel, N. M. (Ed.) (2012). Encyclopedia of Sciences of Learning. New York: Springer.

Sen, A. (1998). Capacidad y bienestar. En M. Nussbaum y A. Sen, La calidad de vida (pp. 54-83). México D. F.: Fondo de Cultura Económica.

Sen, A. (2005). Human Rights and Capabilities. Journal of Human Development, 6(2), 151-66.

Sharples, M. (2000). The design of personal mobile technologies for lifelong learning. Computers \& Education, 34, 177-193.

Shum, S. J. B., y Luckin, R. (Eds.) (2019). Learning analytics and AI: Politics, pedagogy and practices. British Journal of Educational Technology, 50(6), 2785-2793.

Steghofer, J.-Ph. (2017). The Next Generation of Socio-Technical Systems. Human Impacts, Magazine Articles, Societal Impact, September 29th, 2017.

Terras, M. M. y Ramsay, J. (2015). Massive open online courses (MOOCs): Insights and challenges from a psychological perspective. British Journal of Educational Technology, 46(3), Special Issue: Massive Open Online Courses (MOOCs), 472-487.

Touriñán, J. M. (2004). Interculturalismo, globalidad y localidad: estrategias de encuentro para la educación. Bordón, 56(1), 25-47.

Touriñán, J. M. (2019). "Dónde está la educación", Educación para el desarrollo, a propósito de los derechos de la cuarta generación. Recuperado de http://dondestalaeducacion.com/conceptos/conceptos2.html

Unesco (2019). Social and Human Sciences. Poverty. (Recuperado: 19 de noviembre de 2019).

Vázquez, G. (1993). Inteligencia, tecnología y escuela en la sociedad post-industrial. En McClintock, R., Streibel, M. y Vázquez, G., Comunicación, tecnología y diseños de instrucción. La construcción del conocimiento escolar y el uso de los ordenadores (pp. 207-252). Madrid: MEC-CIDE.

Vázquez, G. (2002) El sistema educativo ante la educación de calidad para todos a lo largo y ancho de la vida. Revista de Educación, Número extraordinario, $39-57$.

Vázquez, G. (2016). ¿Saber igual a saber hacer? En Grupo SI(e)TE, Educación. Repensar las ideas dominantes en educación (pp. 95-104). Santiago de Compostela: Andavira.

Wakkary, R., Desjardins, A., and Hauser, S. (2015). Unselfconscious Interaction: A Conceptual Construct. Interacting with Computers Advance. Access published July 17, 2015. Published by Oxford University Press on behalf of The British Computer Society. 
Wheelahan, L. (2007). What are the implications of an uncertain future for pedagogy, curriculum and qualifications. En M. Osborne, M. Houston, y N. Toman, The Pedagogy of Lifelong Learning (pp. 143-153). London: Routledge.

Zimmerman, B. J. (2002). Becoming a self-regulated learner: An overview. Theory into Practice, 41(2), 64-70.

Zimmerman, B. J. (2015). Self-Regulated Learning: Theories, Measures, and Outcomes. En J. D. Wright (Ed.), International Encyclopedia of the Social and Behavioral Sciences (pp. 541-546). Oxford: Elsevier. 